\title{
Complexity of Hantavirus, its Traits and Treatment
}

\author{
Manu Mitra* \\ Department of Electrical Engineering, Alumnus of University of Bridgeport, USA
}

*Corresponding author: Manu Mitra, Department of Electrical Engineering, Alumnus of University of Bridgeport, USA.

To Cite This Article: Manu Mitra. Complexity of Hantavirus, its Traits and Treatment. Am J Biomed Sci \& Res. 2019 - 2(6). AJBSR.MS.ID.000620. DOI: 10.34297/AJBSR.2019.02.000620

Received: April 10, 2019 | Published: May 08, 2019

Introduction

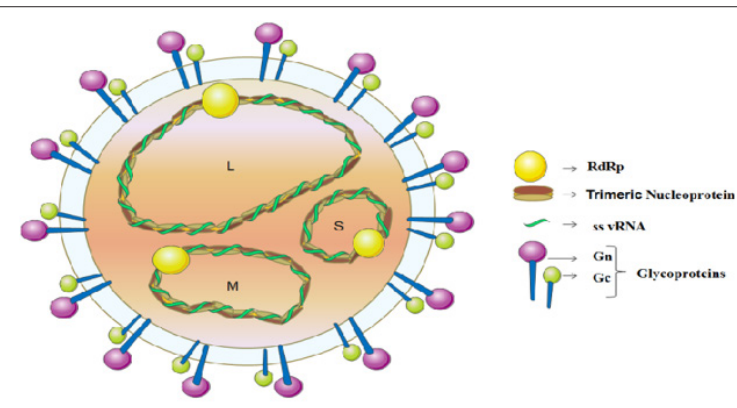

Figure 1: Illustrates Hantavirus genes and proteins. Schematic representation of the virion, containing the RdRp (RNA-dependent RNA polymerase), (-) ss viral RNA (vRNA), Trimeric Nucleoprotein and Spike complex glycoproteins (Gn and Gc). Image Credit: Semantics Scholar [4].

Hantavirus is also called as orthohantavirus and are singlestranded, enveloped, negative RNA virus [(-) ss RNA viruses] [1]. It was named after Hantan River area in South Korea where its occurrence was observed and was isolated in 1976 by Karl M. Johnson and Ho-Wang Lee. There are many strains of Hantavirus; normally rodents are the carrier of these virus. Various strains carried by different rodent species are familiar to cause various types of illness in human, most remarkably, Hemorrhagic Fever with Renal Syndrome (HFRS) which was identified during the Korean War - and Hantavirus Pulmonary Syndrome (HPS) which was discovered in 1993 outbreak in the Southwestern United States. Serious HFRS causes acute kidney failure, while HPS gets filling lungs with fluid (edema). Mortality rate of HFRS is 1 to 15 percent while HPS is 38 percent. One of the remarkable outbreaks of Hantavirus in United States was observed of various strains at Yosemite National Park in late 2012 [2,3] (Figure 1-3).

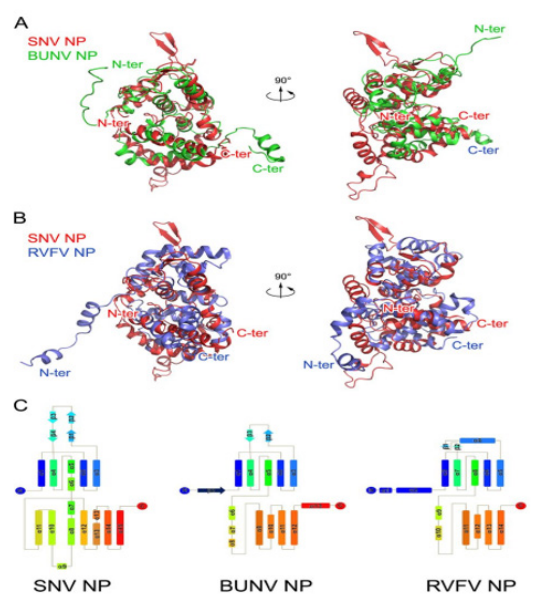

Figure 2: Illustrates Comparison of hantavirus NP with orthobunyavirus and phlebovirus NPs. (A and B) The structures of BUNV (orthobunyavirus) NP (A) and RVFV (phlebovirus) NP (B) are aligned with that of SNV NP, and they are shown in two perpendicular views. Polypeptides of SNV, BUNV, and RVFV NPs are in red, green, and blue, respectively. The N-and C-terminal ends of each molecule are indicated. (C) Topologies of SNV, BUNV, and RVFV NPs. Helices and strands are, respectively, presented as rectangles and arrows and are rainbow colored, where blue and red indicate $\mathrm{N}$ - and C-terminal ends. Image Credit: Journal of Virology [5]. 


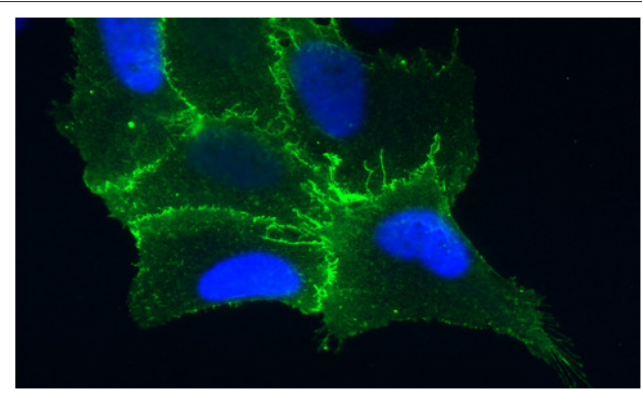

Figure 3: Illustrates cell from the lining of the lung is expressing the protein receptor PCDH1 (in blue), which is found on cell membranes. Image Credit: Albert Einstein College of Medicine [6].

\section{Template for Drug Design from Structure of Hantavirus Protein}

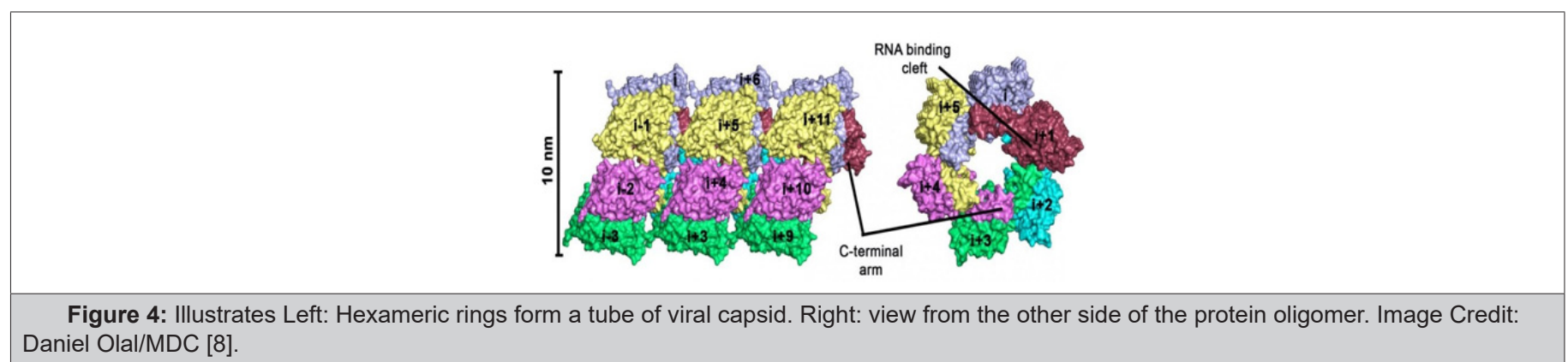

Bank voles are little rodent's family (Myodes glareolus) that are not fatal by themselves, but their excreta may contain one of the dangerous strains of Hantavirus. However, bank voles are not affected by this virus. In central and northern Europe, contamination is followed by fever, headache or also renal failure [4,5]. The strains of Hantavirus that occurs in East Asia is even more fatal; and up to 5 percent of infected patients die from severe respiratory disorders, hemorrhagic fever or renal failures. Dr. Daniel Olal and Prof. Oliver Daumke from Berlin have evaluated nucleoprotein of Hantavirus by novel method of X-ray crystallography and declassified its three dimensional structure. They have worked on how individual nucleoproteins oligomerize in the existence of RNA molecules and hexameric circular complexes. Nucleoprotein plays a very vital role in replication of viral genome [6]. If its function is disrupted, the cell will be unable to develop functional virus elements. Hence this protein is model target structure for the virus. They also identified three binding pockets on the surface of the protein that can take care of docking sites for such compounds. "This structure could be useful for design of small molecules that particularly block nucleoprotein. Technology for drug development is gets better all the time" was confirmed by Olal $[7,8]$ (Figure 4).

\section{DNA Vaccine and Eggs of Ducks May Defend Against Hantavirus}

Army academics with association of other industry collaborators had successfully accomplished in defending laboratory animals from lethal Hantavirus using innovative approach that merges DNA vaccine and duck eggs. As per first author Jay W. Hooper of United States Army Medical Research Institute of Infectious Diseases (USAMRIID), this is the novel method that the DNA vaccine combined with duck egg system has demonstrated to create an antiviral results defending Hantavirus. In this analysis, scientists used a hamster model of Andes virus, which is one of the most vital cause of HPS in South America and Hantavirus was known to be transmitted person to person. Contamination of Syrian hamsters with Andes virus explained the results that disease that closely imitates human HPS in incubation time; syndrome of respiratory, distress and disease pathology. This makes it a prototype system for declassifying the feasibility of post exposure protection strategies. Teaming up with Aldevron of Fargo, N.D. and the Universidad del Desarrollo in Santiago, Chile, Hooper analyzed a natural product, human polyclonal antibody, which was acquired as fresh frozen plasma (FFP) from a patient who sustained HPS.

Their outcome indicates that FFP as a post-exposure preventive remedy for HPS. They also vaccinated ducks with a DNA vaccine against Andes virus. This vaccine uses genetic material or DNA that encodes particular Hantavirus gene to extract an immune response in the receiver. Later they sanitized an antibody called IgY from the yolks of the duck eggs. This sanitized IgY was capable of neutralizing Andes virus when tested in cell culture. Most remarkably, it also shielded Syrian hamsters from deadly HPS even when managed as a single injection survived several days after hamsters had been open to deadly dose of virus. This work indicates the practicability of using DNA vaccine technology coupled with duck egg method. This method could supplement or replace traditional treatment of Hantavirus. Moreover, this new novel method can be scaled as required and eradicated the compulsion of using blood products from HPS sustained patient. "This antivirus production, if fully developed and manufactured; it has the potential to future outbreak situations and it could be used to treat health care employees and others who have close contact with HPS patients" was said by Hooper $[9,10]$. 


\section{Cholesterol Performs a Crucial Role in Hantavirus}

Viruses mutates very fast, that makes it quickly become more resilient to antiviral drugs. It is noteworthy that viruses also depends on proteins and nutrients supplied by their hosts and hence one of the action plan is to identify new antivirus drugs that can identify and target such host cell components. Proteins that participates in the regulation of cholesterol are very imperative for Hantavirus entry into human host cells. Collaborated with team, Paul Bates and Kenneth Briley from the university of Pennsylvania; identified new pathways in human cells there were very essential for Hantavirus contamination. They concentrated on a member of the Hantavirus group called Andes virus (ANDV), they used less dangerous viruses that were genetically engineered to get results and characteristics with ANDV, and then used ANDV itself to confirm that results were correct for Hantavirus as well.

In two independent genetic research, scientists found that four proteins that were participated in Hantavirus contamination and all of them were part of a protein complex that control cholesterol results in mammalian cells. Next they tested an experimental drug that targets one of the four protein could restrict viral entry. They concluded that treating cells that originated from human airways with this drug before revealing them to virus, made the cells less responsive to virus contamination. Because one of the effects of this preliminary drug is that it lowers cholesterol levels in cells. "The sensitivity of ANDV to safe, adequate cholesterol reducing drugs advance new treatments for ANDV contamination and pathogens. Targeting this process may lead to development of effective antivirus" was confirmed by authors $[11,12]$.

\section{Can Statins Used Against Hantavirus?}

Researchers at Perlman School of Medicine and Microbiologist might have found novel method to use statins, the well-known blockbuster cholesterol reducing drugs to defend against Hantavirus. The four proteins analyzed by the researchers are the part of protein complex that controls cholesterol. They found that remedying of human airways cells with a generic statin called mevastatin, which reduces cholesterol by a mechanism that does not participate in four proteins. They made the human airway cells less responsive to ANDV contamination. They tested both preliminary drug called PF-429242 and mevastatin and both were competent against Hantavirus. Although, drug inhibition was initially used the engineered virus. Researchers used ANDV itself in high containment Biosafety level (BSL)-3. This research was conducted at University of Pennsylvania School of Medicine $[13,14]$ (Figure 5).

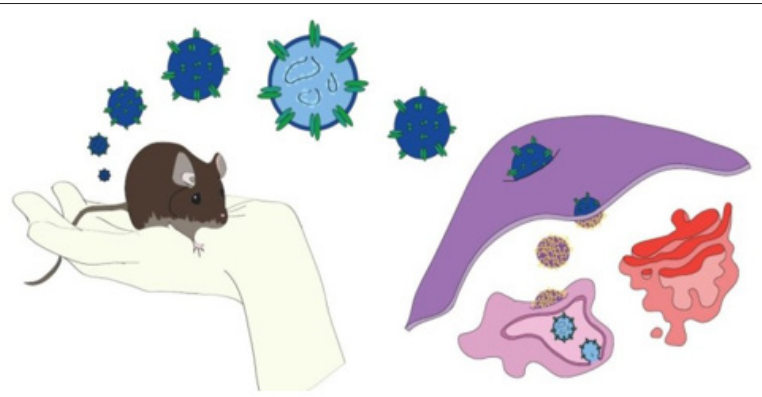

Figure 5: Illustrates Hanta entry path. Image Credit: Hannah Barbian, Courtesy of University of Pennsylvania School of Medicine [14]

\section{Molecular Signature Decoded of Hantavirus in Humans}

Researchers at Charité of Universitätsmedizin Berlin have accomplished in interpreting the molecular signature of Hantavirus that led to an increasing number of outbreaks in Germany. This disease mostly affects kidneys and lungs. In this study shows comparison of the genetic information of a specific viral strain detected in a patient with Hantavirus in Germany. Hantavirus are concealed by specific types of rodents and are transmitted to humans through their excrement. Robert Koch Institute has already registered 2,261 cases of virus in the year 2012. This requires significant research efforts into the illness, its spread and risk of contamination. Through this result of the study, virus break outs and risk of human contamination within Germany can be better defined and understood $[15,16]$.

\section{Acknowledgment}

Author would like to thank Prof. Navarun Gupta, Prof. Hassan Bajwa, Prof. Linfeng Zhang and Prof. Hmurcik for their academic support. Author also thanks anonymous reviewers for their comments.

\section{References}

1. Orthohantavirus (2018) In Wikipedia, the free encyclopedia.

2. Virus (2018) In Wikipedia the free encyclopedia.

3. Marshall J B (2013) 8 Historically Terrifying Viruses.

4. Singh P, Talwar P, Palaniyandi R (2014) Hantavirus Pulmonary Syndrome (HPS): A Concise Review based on Current Knowledge and Emerging Concept. J App Pharm Sci 4(11): 122-130.

5. Guo Y, Wang W, Sun Y, Ma C, Wang X, et al. (2015) Crystal Structure of the Core Region of Hantavirus Nucleocapsid Protein Reveals the Mechanism for Ribonucleoprotein Complex Formation. Journal of Virology 90(2): 1048-1061.

6. Medicalxpress (2018) Study identifies how hantaviruses infect lung cells.

7. Daniel Olal, Oliver Daumke (2016) Structure of the Hantavirus Nucleoprotein Provides Insights into the Mechanism of RNA Encapsidation. Cell Reports.

8. Max Delbrück Center for Molecular Medicine in the Helmholtz Association (2016) Structure of a hantavirus protein as a promising model for drug design: X-ray crystallography provides drug template against disease transmitted by small rodents. Science Daily. 
9. Rebecca Brocato, Matthew Josleyn, John Ballantyne, Pablo Vial, Jay W Hooper (2012) DNA Vaccine-Generated Duck Polyclonal Antibodies as a Postexposure Prophylactic to Prevent Hantavirus Pulmonary Syndrome (HPS). PLoS ONE 7(4): e35996.

10. US Army Medical Research Institute of Infectious Diseases (2012) DNA vaccine and duck eggs protect against hantavirus disease. ScienceDaily.

11. Josiah Petersen, Mary Jane Drake, Emily A Bruce, Amber M Riblett Chukwuka A Didigu, et al. (2014) The Major Cellular Sterol Regulatory Pathway Is Required for Andes Virus Infection. PLoS Pathogens 10(2).

12. PLOS (2014) Cholesterol plays a critical role in hantavirus infection. ScienceDaily.
13. Josiah Petersen, Mary Jane Drake, Emily A Bruce, Amber M Riblett, Chukwuka A Didigu, et al. (2014) The Major Cellular Sterol Regulatory Pathway Is Required for Andes Virus Infection. PLoS Pathogens 10(2).

14. University of Pennsylvania School of Medicine (2014) Could statins be used to fight a deadly viral infection? ScienceDaily.

15. Jakob Ettinger, Jorg Hofmann, Martin Enders, Friedemann Tewald, Rainer M Oehme, et al. (2012) Multiple Synchronous Outbreaks of Puumala Virus. Emerging Infectious Diseases 18(9): 1461.

16. Charité - Universitätsmedizin Berlin (2012) Molecular signature of hantavirus infection in humans decoded. ScienceDaily. 